\title{
Are There Resilience Resources That Can Be Harnessed To Manage Psychological Distress In Spanish-speaking Latinas With Breast Cancer?
}

\author{
Jasmine Santoyo-Olsson, ${ }^{1}$ Carmen Ortiz, ${ }^{2}$ Anita L. Stewart, ${ }^{1}$ Anna Maria Nápoles ${ }^{3}$
}

1 University of California San Francisco, ${ }^{2}$ Círculo de Vida Cancer Support and Resource Center, ${ }^{3}$ National Institute on Minority Health and Health Disparities, NIH

Background: Latina breast cancer survivors (LBCS) are at higher risk of psychological distress than their white counterparts. Adapting to cancer is influenced by intrapersonal, interpersonal, and environmental factors, which may enhance resilience. Little is known about how resilience resources affects health in this population.

Aims: Examine the extent to which intrapersonal and interpersonal resilience resources are associated with psychological distress among Spanish-speaking LBCS in the first year of survivorship.

Methods: Cross-sectional analysis of baseline data from a 6-month RCT testing a peer-delivered cognitive behavioral stress management intervention for Spanish-speaking LBCS. Community-based recruiters identified potential participants from public hospital cancer centers or cancer support organizations who were: 1) Spanish-speaking Latinas; 2) diagnosed with non-metastatic breast cancer in past year; and 3) living in 5 San Francisco Bay Area counties. Linear regression analyses examined bivariate and multivariate associations of resources with health distress and anxiety. We included resilience resources in multivariate, if associated significantly with health distress or anxiety at $p<.20$ in bivariate. Final model regressed health distress or anxiety on resilience resources, controlling for demographic/clinical characteristics.

Results: All resilience resources were associated significantly with health distress and anxiety in bivariate. Self-efficacy for coping with breast cancer treatment was associated independently and inversely with both health distress $(p<0.05)$ and anxiety $(p<0.05)$. Coping confidence with general problems $(p<0.05)$ and having a sense of neighborhood cohesion $(p<0.05)$ were associated independently and inversely with health distress. Feeling a sense of peace $(p<0.05)$ and having tangible social support $(p<0.05)$ were associated independently and inversely with anxiety.

Conceptual Framework of Resilience Resources for Managing Psychological Distress
Conclusions: Self-efficacy for coping with breast cancer treatment, coping confidence, and having a sense of neighborhood cohesion were associated independently and inversely with health distress. Feeling a sense of peace and having tangible support were associated independently and inversely with anxiety. Interventions that aim to reduce health distress and anxiety among LBCS can focus on skills that capitalize on these resilience resources.

Results: Sample Characteristics ( $N=151)$

- Mean age in years=50; range (28-81)

- 68\% Mexican, 23\% Central American, 9\% South American

- $84 \%$ had high school or less than high school education

- $>80 \%$ enrolled within 6 months of diagnosis

- $44 \%$ had mastectomy

- $40 \%$ had radiation + chemotherapy

- Mean health distress $(1-5)^{*}=3.14(S D=1.03)$

- Mean anxiety $(0-4)^{*}=0.97(\mathrm{SD}=0.86)$

\begin{tabular}{|c|c|c|}
\hline \multicolumn{3}{|c|}{$\begin{array}{c}\text { Results: Effects of Resilience Resources on } \\
\text { Psychological Distress }\end{array}$} \\
\hline & Health Distress & Anxiety \\
\hline & $\begin{array}{l}\infty \text { Adjusted } \\
\beta \text { (p-value) }\end{array}$ & $\begin{array}{l}\infty \text { Adjusted } \\
\beta \text { (p-value) }\end{array}$ \\
\hline & $R^{2}=0.34$ & $R^{2}=0.32$ \\
\hline \multicolumn{3}{|l|}{ Intrapersonal } \\
\hline $\begin{array}{l}\text { Coping with breast } \\
\text { cancer treatment }\end{array}$ & $-0.10(<.05)$ & $-0.11(<.05)$ \\
\hline Meaning/peace & $-0.13(0.42)$ & $-0.30(<.05)$ \\
\hline Coping confidence & $-0.32(<.05)$ & $-0.10(0.32)$ \\
\hline \multicolumn{3}{|l|}{ Interpersonal } \\
\hline $\begin{array}{l}\text { Neighborhood } \\
\text { cohesion }\end{array}$ & $-0.16(<.05)$ & $-0.08(0.24)$ \\
\hline Tangible support & $-0.14(0.08)$ & $-0.15(<.05)$ \\
\hline
\end{tabular}
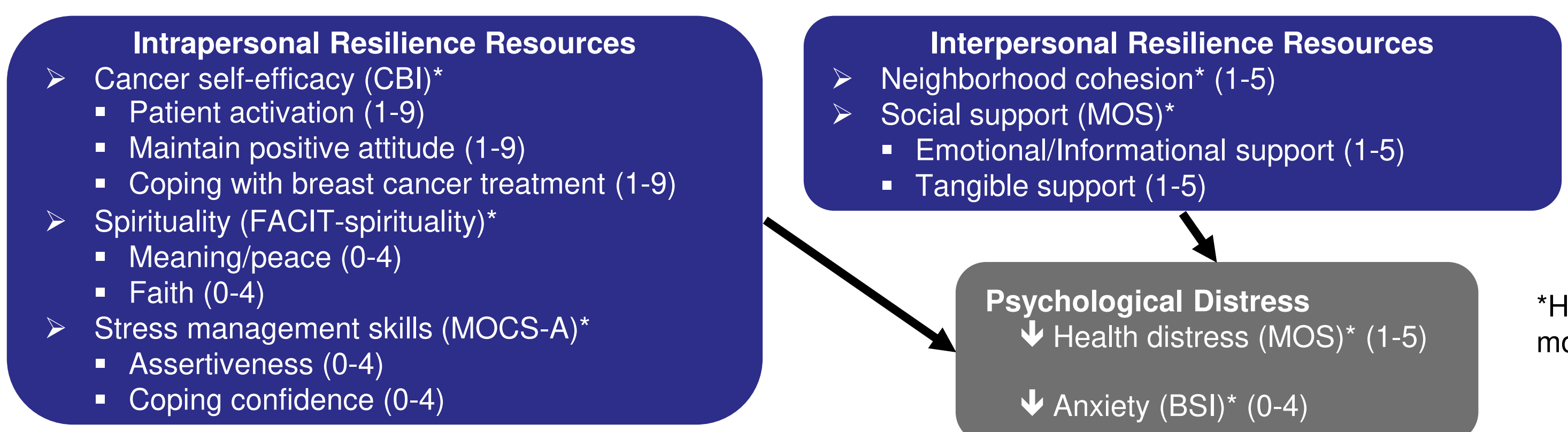